\title{
2017—venture funding goes into overdrive
}

\section{Laura DeFrancesco}

Although biotech stocks nose-dived in the last quarter, the NASDAQ Biotech Index still returned a double-digit increase year on year. Compared with 2016, mergers and acquisition were down (just 54 in total), follow-on financings were up and initial public offerings

\section{Stock market performance}

The biotech index had a volatile year; mediocre earnings and Celgene's disappointing mongersen trial sent shares spiraling in the last quarter.

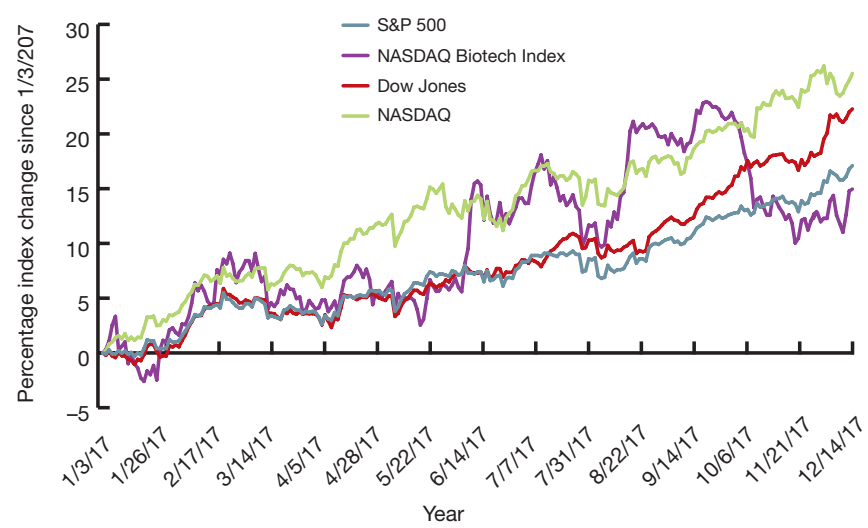

\section{Global biotech initial public offerings}

Fewer floats seen on European and Asia-Pacific markets; biotech raised more on US markets.

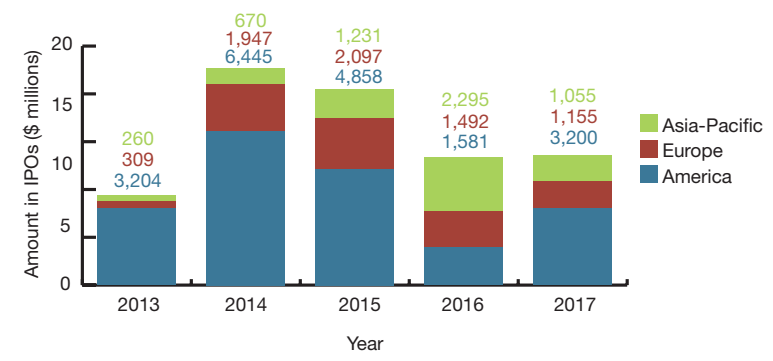

\begin{tabular}{lccccc} 
& $\mathbf{2 0 1 3}$ & $\mathbf{2 0 1 4}$ & $\mathbf{2 0 1 5}$ & $\mathbf{2 0 1 6}$ & $\mathbf{2 0 1 7}$ \\
\hline Americas & 40 & 74 & 50 & 26 & 32 \\
Europe & 10 & 35 & 40 & 25 & 26 \\
Asia-Pacific & 9 & 8 & 6 & 23 & 13 \\
\hline
\end{tabular}

\section{Global biotech venture capital investment}

The influx of venture capital was a continued bright point, approaching $\$ 16$ billion.

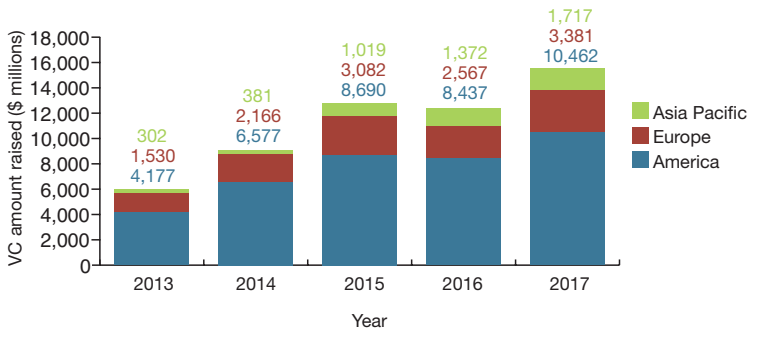

\begin{tabular}{lccccc} 
& $\mathbf{2 0 1 3}$ & $\mathbf{2 0 1 4}$ & $\mathbf{2 0 1 5}$ & $\mathbf{2 0 1 6}$ & $\mathbf{2 0 1 7}$ \\
\hline Americas & 23 & 30 & 34 & 37 & 45 \\
Europe & 137 & 138 & 139 & 132 & 125 \\
Asia-Pacific & 274 & 300 & 327 & 275 & 333 \\
\hline \multicolumn{2}{l}{ Source: BCIQ: BioCentury Online Intelligence. }
\end{tabular}

Laura DeFrancesco is a Senior Editor at Nature Biotechnology.
(IPOs) raised similar totals in funding. But it was venture capital investment that continued to gorge the sector, with a few companies raising eye-popping amounts-all in all, the sector raised just short of $\$ 16$ billion in risk capital.

\section{Global biotech industry financing}

Though IPOs and debt financing were down, biotechs raised a healthy $\$ 75$ billion, excluding partnerships.

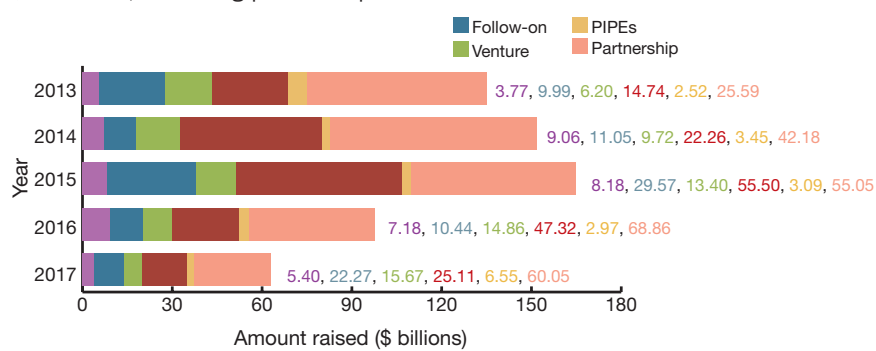

PIPEs, private ivestment in public equity. Source: BCIQ: BioCentury Online Intelligence.

Notable 2017 deals

IPOs

\begin{tabular}{|c|c|c|c|}
\hline Company (lead underwriters) & $\begin{array}{l}\text { Amount raised } \\
(\$ \text { millions })\end{array}$ & $\begin{array}{c}\text { Date } \\
\text { completed }\end{array}$ & $\begin{array}{r}\text { Change in sto } \\
\text { since offer }( \\
1 / 12 / 20 \\
\end{array}$ \\
\hline $\begin{array}{l}\text { WuXi Biologics (BofA Merrill Lynch, Morgan Stanley, } \\
\text { China Merchants Securities) }\end{array}$ & 585 & 7-Jun & \\
\hline $\begin{array}{l}\text { Denali (Goldman Sachs, Morgan Stanley, JP Morgan, } \\
\text { Evercore ISI) }\end{array}$ & 287 & 7-Dec & \\
\hline $\begin{array}{l}\text { BioHaven Pharma (Morgan Stanley, Piper Jaffray, } \\
\text { Barclays Capital, William Blair, Needham }\end{array}$ & 194 & 4-May & \\
\hline TissueGene (NH Investment \& Securities) & 182 & 6-Nov & \\
\hline ZaiLab (JP Morgan, Citigroup, Leerink Partners) & 172 & 20-Sep & \\
\hline
\end{tabular}

Venture capital

\begin{tabular}{|c|c|c|c|}
\hline Company & $\begin{array}{c}\text { Amount raised } \\
\text { (\$ millions) }\end{array}$ & $\begin{array}{c}\text { Round } \\
\text { number }\end{array}$ & Date closed \\
\hline $\begin{array}{l}\text { Grail (Arch Venture Partners, Johnson \& Johnson } \\
\text { Innovation, Amazon and others) }\end{array}$ & 900 & 2 & 1-Mar \\
\hline $\begin{array}{l}\text { Guardant Health (Softbank, T. Rowe Price, Temasek, } \\
\text { Sequoia Capital, Khosla Ventures, Lightspeed Venture } \\
\text { Partners, OrbiMed Advisors, 8VC) }\end{array}$ & 360 & 5 & 11-May \\
\hline $\begin{array}{l}\text { Ginkgo Bioworks (Existing investors, Viking Global Investors } \\
\text { LP, Y Combinator, Cascade Investment, William Gates III, } \\
\text { General Atlantic, and other undisclosed investors) }\end{array}$ & 275 & 4 & 14-Dec \\
\hline $\begin{array}{l}\text { Harmony Biosciences (Valor Equity Partners, Fidelity, HBM } \\
\text { Healthcare Investments, Vivo Capital, venBio LLC, Novo } \\
\text { Holdings A/S, Nan Fung) }\end{array}$ & 270 & ND & $6-0 c t$ \\
\hline 23andme (Sequoia Capital) & 250 & ND & 12-Sep \\
\hline
\end{tabular}

Mergers and acquisitions

\begin{tabular}{|c|c|c|c|c|}
\hline \multicolumn{2}{|l|}{ Target } & Acquirer & Value (\$ millions) & Date announced \\
\hline \multicolumn{2}{|l|}{ Actelion } & Johnson \& Johnsor & 30,000 & 26-Jan \\
\hline \multicolumn{2}{|l|}{ Kite Pharma } & Gilead & 11,900 & 28-Aug \\
\hline \multicolumn{2}{|l|}{ Pantheon } & Thermo Fisher & 7,200 & 15-May \\
\hline \multicolumn{2}{|l|}{ Ariad } & Takeda & 5,200 & 9-Jan \\
\hline \multicolumn{2}{|l|}{ Fresenius } & Akorn & 4,300 & 24-May \\
\hline \multicolumn{5}{|c|}{ Licensing/collaboration } \\
\hline Researcher & Investor & \multicolumn{3}{|c|}{ Value (\$ millions) Deal description } \\
\hline AstraZeneca & Merck \& Co. & $\begin{array}{ll}8,500 & c \\
& a \\
& \text { ti }\end{array}$ & \multicolumn{2}{|c|}{$\begin{array}{l}\text { Codevelop small-molecule PARP inhibitor Lynparza } \\
\text { and MEK inhibitor selumetinib to treat multiple } \\
\text { tumor types }\end{array}$} \\
\hline $\begin{array}{l}\text { Assembly } \\
\text { Biosciences }\end{array}$ & Allergan & 2,830 & \multicolumn{2}{|c|}{$\begin{array}{l}\text { Exclusive, worldwide rights to orally delivered, micro- } \\
\text { biome-derived mixtures of live biotherapeutic products } \\
\text { (ABI-M2O1 and ABI-M301) and two undisclosed } \\
\text { compounds to treat irritable bowel syndrome }\end{array}$} \\
\hline Ablynx & Sanofi & 2,735 & \multicolumn{2}{|c|}{$\begin{array}{l}\text { Exclusive, worldwide rights to eight multi-specific } \\
\text { nanobodies to treat immune-mediated inflammatory } \\
\text { diseases }\end{array}$} \\
\hline $\begin{array}{l}\text { Pieris } \\
\text { Pharmaceuticals }\end{array}$ & AstraZeneca & 2,157 & \multicolumn{2}{|c|}{$\begin{array}{l}\text { Worldwide rights to develop and commercialize five } \\
\text { inhaled respiratory disease candidates, including } \\
\text { preclinical asthma candidate PRS-060 }\end{array}$} \\
\hline Halozyme & $\begin{array}{l}\text { Bristol-Myers } \\
\text { Squibb }\end{array}$ & 1,865 & \multicolumn{2}{|c|}{$\begin{array}{l}\text { Rights to use its recombinant human PH2O hyal- } \\
\text { uronidase delivery technology to develop subcutane- } \\
\text { ous versions of immuno-oncology products against } \\
\text { up to } 11 \text { targets }\end{array}$} \\
\hline
\end{tabular}

